\title{
Absence of telomerase reverse transcriptase promoter mutations in neuroblastoma
}

\author{
SVEN LINDNER $^{1-3}$, HAGEN S. BACHMANN ${ }^{4}$, ANDREA ODERSKY ${ }^{1}$, SIMON SCHAEFERS $^{1-3}$, \\ LUDGER KLEIN-HITPASS ${ }^{5}$, BARBARA HERO ${ }^{6}$, MATTHIAS FISCHER ${ }^{6,7}$, ANGELIKA EGGERT ${ }^{8}$, \\ ALEXANDER SCHRAMM $^{1}$ and JOHANNES H. SCHULTE ${ }^{1-3,9,10}$
}

\author{
${ }^{1}$ Department of Pediatric Oncology and Hematology, University Children's Hospital Essen; \\ ${ }^{2}$ German Consortium for Translational Cancer Research (DKTK), Partner Site Essen/Duesseldorf, D-45122 Essen; \\ ${ }^{3}$ German Cancer Research Center (DKFZ), D-69120 Heidelberg; ${ }^{4}$ Institute of Pharmacogenetics, University Hospital Essen, \\ D-45147 Essen; ${ }^{5}$ Institute of Cell Biology (Cancer Research), Faculty of Medicine, University of Duisburg-Essen, \\ D-45122 Essen; ${ }^{6}$ Department of Pediatric Oncology and Hematology, University Children's Hospital of Cologne, \\ D-50924 Cologne; ${ }^{7}$ Center for Molecular Medicine Cologne (CMMC), University of Cologne, D-50931 Cologne; \\ ${ }^{8}$ Department of Pediatric Oncology/Hematology, Charité-Universitätsmedizin Berlin, D-10117 Berlin; \\ ${ }^{9}$ Translational Neuro-Oncology, West German Cancer Center (WTZ), University Hospital Essen; \\ ${ }^{10}$ Centre for Medical Biotechnology, University Duisburg-Essen, D-45147 Essen, Germany
}

Received March 13, 2015; Accepted April 22, 2015

DOI: $10.3892 /$ br.2015.463

\begin{abstract}
Maintenance of telomere length is a critical hallmark of malignant transformation. While silenced in somatic cells, telomerase reverse transcriptase (TERT), the catalytic subunit of telomerase, is frequently overexpressed in malignant cells thereby maintaining their telomere length. Specific point mutations in the TERT promoter region have recently been identified in melanoma and other tumor entities resulting in high TERT expression. Neuroblastoma is the most common extracranial tumor of childhood, arising from neural-crest progenitor cells. TERT overexpression has been observed in the majority of neuroblastoma. Taking into consideration that TERT promoter mutations are frequently described in neural-crest-derived tumors such as melanoma, as well as a variety of other neuronal tumors, the present study analyzed the frequency of TERT promoter mutations in primary neuroblastoma and neuroblastoma cell lines. In 131 neuroblastoma primary tumors representing the whole spectrum of neuroblastoma, no TERT promoter mutations were detected. However, in 3 out of 19 neuroblastoma cell lines the previously described C228T TERT promoter mutation was present. In conclusion, the TERT promoter mutations are not a frequent mechanism of TERT overexpression in neuroblastoma.
\end{abstract}

Correspondence to: Dr Sven Lindner, Department of Pediatric Oncology and Haematology, University Children's Hospital Essen, Hufelandstrasse 55, D-45122 Essen, Germany

E-mail: sven.lindner@rub.de

Key words: neuroblastoma, telomerase reverse transcriptase, alternative lengthening of telomeres, telomeres, sequencing

\section{Introduction}

Each mitotic cell division results in the shortening of telomeres (1). In somatic cells, subsequent shortening of telomeres eventually leads to senescence. The multiunit enzyme telomerase maintains telomere length by adding repetitive sequences to telomeres at each replicative cycle. However, the telomerase activity is absent in somatic cells. By contrast, malignant cells (re-)express telomerase or use an alternative mechanism to maintain telomere length, known as alternative lengthening of telomeres (ALT) (2).

Telomerase reverse transcriptase (TERT) is an essential part of the telomerase enzyme complex (3). Most recently, specific point mutations were described in the promoter region of the TERT gene in familial and sporadic melanoma (4). These point mutations lead to a cytosine to thymine exchange at the positions 228 or 250 upstream of the start codon of TERT, further referred to as C228T and C250T, respectively (3-5). Through these mutations, the TERT promoter acquires novel binding sites for the E twenty-six transcription factor, resulting in increased TERT expression (6). Subsequently, TERT promoter mutations were described in a variety of tumors, including several neuronal tumors as medulloblastoma (7) and glioma, as well as other tumor entities $(4,5)$. Of note, the presence of TERT promoter mutations often correlated with aggressive disease and an adverse outcome.

Neuroblastoma is the most common extracranial tumor during childhood arising from the neural-crest $(8,9)$. Only few recurrent mutations or genomic rearrangements have been described thus far (10). Among these are the genomic amplifications of $M Y C N$ and activating mutations of anaplastic lymphoma kinase. Most recently, two independent studies described ALT through mutations in the transcriptional 
regulator $\alpha$-thalassemia/mental retardation $\mathrm{X}$-linked (ATRX) in a minor fraction of neuroblastomas $(11,12)$. Although the ALT phenotype may not be limited to neuroblastomas with $A T R X$ mutations, the majority of neuroblastomas express telomerase and in particular TERT (13). Of note, high TERT expression was detected in $M Y C N$-amplified tumors, but even in $M Y C N$ single copy neuroblastomas a high TERT expression correlated with adverse outcome (13). By contrast, low or absent TERT expression was detected in favorable neuroblastomas and absence of telomerase activity has been proposed as a potential mechanism to explain the high rate of spontaneous regression of favorable neuroblastomas (14). While MYCN is known to induce TERT transcription $(15,16)$ and TERT amplification has been described in few neuroblastoma cases (17), the mechanism that drives TERT expression remains elusive in the majority of neuroblastomas.

As TERT promoter mutations are frequent in neural-crest-derived melanoma, as well as in various neuronal tumors including medulloblastoma, the present study analyzed the frequency of TERT promoter mutations in neuroblastoma.

\section{Materials and methods}

Primary tumor samples. Primary tumor samples and patient data were obtained from the German Society of Pediatric Oncology and Hematology Tumor Bank and Neuroblastoma Study Center (Cologne, Germany), respectively. All the patients were registered with the German neuroblastoma study and written informed consent was obtained. A tumor content of $\geq 60 \%$ was confirmed by a pathologist. DNA was isolated from $\sim 20 \mathrm{mg}$ of snap-frozen tissue obtained prior to cytotoxic treatment using the Puregene Blood Core kit B (Qiagen, Hilden, Germany), according to the manufacturer's instructions.

Cell culture. Neuroblastoma cell lines BEC(2), CHP134, IMR-32, IMR-5-75, KCN, KCN(R), Kelly, LAN1, LAN5, LAN6, NGP, NLF, SH-EP, SH-SY5Y, SK-N-AS, SK-N-BE, SK-N-FI and SK-N-SH were cultured in RPMI-1640 supplemented with $10 \%$ fetal calf serum, $5 \%$ penicillin $(100 \mathrm{U} / \mathrm{ml})$ and streptomycin $(100 \mu \mathrm{g} / \mathrm{ml})$ until reaching a confluence of $70 \%$. Cells were collected and DNA was isolated using the QIAamp DNA kit (Qiagen). All the cell lines were authenticated by short tandem repeat genotyping performed by DSMZ (Braunschweig, Germany) or IDEXX GmbH (Ludwigsburg, Germany).

Polymerase chain reaction (PCR) and sequencing. DNA (100 ng) was used for the PCR reaction. To each PCR reaction, TERT forward (ACGAACGTGGCCAGCGGCAG) and reverse primers (CTGGCGTCCCTGCACCCTGG) were added to amplify a 474-base pair (bp) long region of the TERT promoter (390-bp upstream and 80-bp downstream of the start codon). The PCR program used was as follows: $95^{\circ} \mathrm{C}$ for $180 \mathrm{sec}$, followed by $95^{\circ} \mathrm{C}$ for $30 \mathrm{sec}, 62^{\circ} \mathrm{C}$ for $45 \mathrm{sec}$ and $72^{\circ} \mathrm{C}$ for $60 \mathrm{sec}$ repeated 35 times, and a final amplification step at $72^{\circ} \mathrm{C}$ for $600 \mathrm{sec}$. Sanger sequencing was performed by Sequence Laboratories Göttingen GmbH (Göttingen, Germany).
Table I. Patient data of the 131 sequenced primary neuroblastomas tumors.

\begin{tabular}{lc}
\hline Characteristics & Values, $\mathrm{n}$ \\
\hline Patients & 131 \\
Gender & \\
Female & 47 \\
Male & 84 \\
Stage (INSS) & \\
1 & 29 \\
$2 \mathrm{a}$ & 12 \\
$2 \mathrm{~b}$ & 7 \\
3 & 19 \\
4 & 50 \\
4 S & 14 \\
MYCN status & \\
Non-amplified & 96 \\
Amplified & 33 \\
Not detectable & 2 \\
1p36 status & \\
Wild-type & 90 \\
Deletion & 24 \\
Imbalance & 12 \\
Not detectable & 5 \\
Age at diagnosis, years & \\
$<1$ & 36 \\
1-2 & \\
$>2$ & 50 \\
&
\end{tabular}

INSS, International Neuroblastoma Staging System.

Reverse transcription-quantitative PCR (RT-qPCR). Total RNA was isolated from cells using the RNeasyMini kit (Qiagen) and cDNA synthesis was performed using the SuperScript ${ }^{\circledR}$ RT kit (Invitrogen Life Technologies, Darmstadt, Germany). TERT expression was monitored using the QuantiTect Primer Assay ${ }^{\mathrm{TM}}$ (Qiagen). Expression values were normalized to the geometric mean of GAPDH (18). Data analysis and error propagation were performed using the qbasePLUS software version 1.5 (http://www.biogazelle. com).

Pyrosequencing. To determine the presence of the mutant C228T allele or the wild-type allele using pyrosequencing, the following primers were used to amplify a 151-bp amplicon: Biotinylated forward primer 5'-BIOTIN-CTTCACCTTCCA GCTCCGC-3', and reverse primer 5'-CGCTGCCTGAAA CTCGC-3'. The PCR products were analyzed by pyrosequencing using the primer 5'-GAGGGGCTGGGAGGG CCC-3', on the PyroMark Q96 MD system according to the manufacturer's instructions (Qiagen) and as previously described (19). Results were analyzed using PyroMark MD 1.0 software (Biotage, Uppsala, Sweden).

Genome-wide single-nucleotide polymorphism (SNP) analysis. SNP array experiments were performed according to the standard protocol for Affymetrix CytoScanHD arrays 
Table II. Location of the Affymetrix CytoScanHD array probes located within or near the TERT gene. The probes lying within the TERT gene showed a loss of heterozygousity for SH-EP, SH-SY5Y and SK-N-SH. Probes lying outside of the TERT gene showed a heterozygous status.

\begin{tabular}{lccccc}
\hline & & \multicolumn{3}{c}{ Zygosity status } & \\
\cline { 3 - 4 } Probe ID & Probe start position & SK-N-SH & SH-EP & SH-SY5Y & Comment \\
\hline S-4ELKX & 1240103 & Heterozygous & Heterozygous & Heterozygous & Probe downstream of $T E R T$ gene \\
S-4OOCR & 1267356 & Homozygous & Homozygous & Homozygous & Probe inside of $T E R T$ gene \\
S-4FACZ & 1269006 & Homozygous & Homozygous & Homozygous & Probe inside of $T E R T$ gene \\
S-4IBBK & 1269365 & Homozygous & Homozygous & Homozygous & Probe inside of $T E R T$ gene \\
S-3MNHV & 1299087 & Heterozygous & Heterozygous & Heterozygous & Probe upstream of $T E R T$ gene \\
\hline
\end{tabular}

Chromosomal position of TERT: Chr.5.1253167-1295047; Chromosomal position of the C228T mutation: Chr.5.1295228. TERT, telomerase reverse transcriptase.
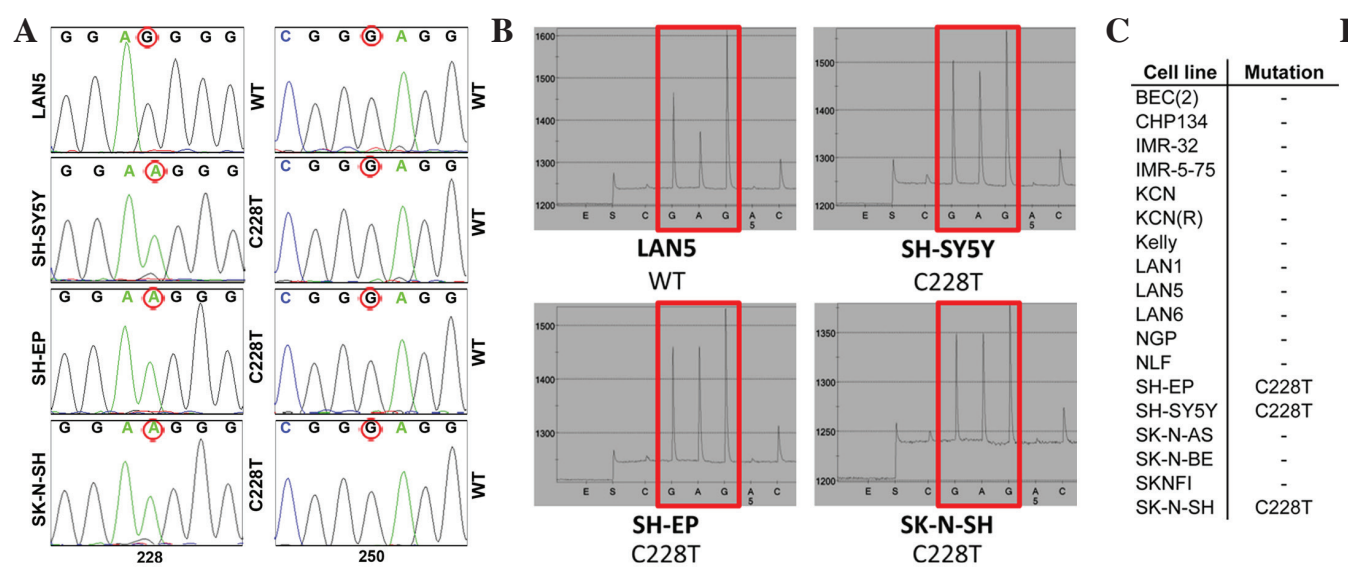

D

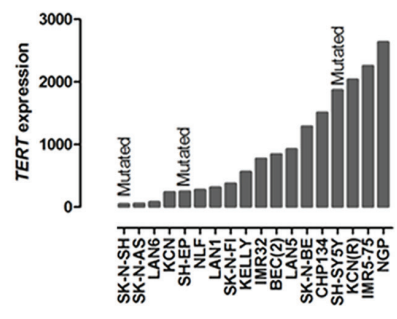

Figure 1. (A) Electropherogram examples of the cell lines SH-SY5Y, SH-EP and SK-N-SH harboring the C228T nucleotide exchange. By contrast, LAN5 shows the wild-type (WT) sequence at the mutation hotspots. (B) Representative pyrograms obtained for WT LAN5 cells, as well as SK-N-SH, SH-EP and SH-SY5Y cells, harboring the C228T telomerase reverse transcriptase (TERT) promoter mutation. Pyrograms were generated by sequential addition of the single-nucleotide polymorphism-specific nucleotides shown beneath each trace. Note that each peak corresponds to one or more identical bases added to the synthesized DNA-strand and that the height of each peak is correlated to the number of sequential nucleotides of the same type. (C) List of the neuroblastoma cell lines sequenced for TERT promoter mutations (C228T=guanine to adenine nucleotide exchange upstream of the TERT promoter at position-228). (D) TERT expression scaled to the lowest expression found in SK-N-SH (TERT expression=1). The cell lines that carry a TERT promoter mutation are marked with 'mutated'.

(Affymetrix, Inc., Santa Clara, CA, USA). In brief, a 250-ng sample of genomic DNA was digested with NspI, ligated to adaptors, amplified by PCR, fragmented and biotin-labeled. The labeled samples were hybridized to Affymetrix CytoscanHD arrays, followed by washing, staining and scanning in the Affymetrix GeneChip Scanner 3000. Analysis was performed using the Affymetrix Chromosome Analysis Suite v2.1.

\section{Results}

TERT promoter mutations are absent in primary neuroblastomas. To determine the frequency of TERT promoter mutations in neuroblastoma, including the previously described C228T and $\mathrm{C} 250 \mathrm{~T}$ mutations, the respective TERT promoter region of 131 primary neuroblastomas was sequenced. These tumors represented the whole spectrum of neuroblastoma regarding stage, MYCN and 1p36 status (Table I). Of note, none of the analyzed neuroblastomas harbored a C228T or a C250T mutation in the TERT promoter region.
TERT promoter mutations in neuroblastoma cell lines. In addition to primary neuroblastomas, 19 neuroblastoma cell lines were analyzed. In 3 out of 19 analyzed cell lines (16\%) a mutation of the TERT promoter was detected. The neuroblastoma cell lines SK-N-SH, SH-SY5Y and SH-EP harbor the C228T mutation (Fig. 1A-C). Of note, SH-SY5Y and SH-EP are subclones of SK-N-SH (20). SK-N-SH was established from a bone marrow biopsy of a 4-year-old girl $(21,22)$. The presence of the C228T TERT promoter mutation in SK-N-SH, SH-SY5Y and SH-EP was independently validated using pyrosequencing (Fig. 1B). As the C228T mutation appeared to be homozygous in SK-N-SH, SH-SY5Y and SH-EP, Affymetrix Human SNP array 6.0 arrays were used to analyze zygosity. The 3 probes located within the TERT gene were homozygeous in all three cell lines, which could point to the presence of a small loss-of-heterozygosity region spanning the TERT gene (Table II). To analyze if the mutations in the TERT promoter correlate with increased TERT expression, the expression was analyzed in all the neuroblastoma cell lines using RT-qPCR. No correlation between mutations and TERT expression was detected (Fig. 1D). 


\section{Discussion}

Analysis of the TERT gene promotor region in 20 neuroblastoma cell lines and 131 primary neuroblastoma tumors revealed mutations in 3 cell lines and no mutations in primary tumors.

Of note, the only 3 mutated cell lines, which harbor the C228T mutation, are SK-N-SH and its 2 subclones SH-EP and $\mathrm{SH}-\mathrm{SY} 5 \mathrm{Y}$, further limiting the general indication of this finding. It is uncommon that TERT mutations occur homozygously, as detected in SK-N-SH, SH-EP and SY5Y. However, further analysis confirmed a loss-of-heterozygosity region in the region of the TERT gene, pointing to a hemizygous rather than a homozygous status of the C228T mutation in these cases. Taking into consideration the variable levels of TERT expression in SK-N-SH, SH-EP and SH-SY5Y cells, the functional indication of the C228T mutations in these cell lines remain questionable.

The absence of TERT mutations in primary neuroblastomas and the majority of analyzed cell lines is unexpected, as TERT expression is of pathogenic relevance in the majority of neuroblastomas (13) and TERT mutations are frequent in related neuroectodermal tumors, such as melanoma and medulloblastoma $(4,7,23,24)$. However, the present results are in line with previous studies by Papathomas et al (25), who did not find TERT promoter mutations in 15 primary neuroblastomas and Killela et al (23), who identified only 2 TERT promoter mutations in 22 neuroblastomas.

The mechanisms by which TERT expression is induced, particularly in $M Y C N$ non-amplified high-risk neuroblastomas, remains elusive and warrants further studies. Potential alternative mechanisms underlying TERT induction in neuroblastoma include epigenetic changes, as well as mutations in more distant regulatory elements that were not analyzed in the present study.

Taken together, we conclude that TERT (core) promoter mutations are not relevant events in neuroblastoma pathogenesis.

\section{Acknowledgements}

The authors acknowledge the funding from the German Ministry for Education and Research (e:MED grant nos. SMOOSE FKZ: 01ZX1303B and SYSMED-NB FKZ: 01ZX1307E to J.H.S.) and the German Cancer Aid (grant no. 111301 to J.H.S.).

\section{References}

1. Shay JW and Wright WE: Role of telomeres and telomerase in cancer. Semin Cancer Biol 21: 349-353, 2011

2. Nabetani A and Ishikawa F: Alternative lengthening of telomeres pathway: Recombination-mediated telomere maintenance mechanism in human cells. J Biochem 149: 5-14, 2011.

3. Griewank KG, Murali R, Schilling B, Scholz S, Sucker A, Song M, Süsskind D, Grabellus F, Zimmer L, Hillen U, et al: TERT promoter mutations in ocular melanoma distinguish between conjunctival and uveal tumours. Br J Cancer 109: 497-501, 2013

4. Horn S, Figl A, Rachakonda PS, Fischer C, Sucker A, Gast A, Kadel S, Moll I, Nagore E, Hemminki K, et al: TERT promoter mutations in familial and sporadic melanoma. Science 339: 959-961, 2013

5. Huang FW, Hodis E, Xu MJ, Kryukov GV, Chin L and Garraway LA: Highly recurrent TERT promoter mutations in human melanoma. Science 339: 957-959, 2013.
6. Xu D, Dwyer J, Li H, Duan W and Liu JP: Ets2 maintains hTERT gene expression and breast cancer cell proliferation by interacting with c-Myc. J Biol Chem 283: 23567-23580, 2008.

7. Remke M, Ramaswamy V, Peacock J, Shih DJ, Koelsche C, Northcott PA, Hill N, Cavalli FM, Kool M, Wang X, et al: TERT promoter mutations are highly recurrent in SHH subgroup medulloblastoma. Acta Neuropathol 126: 917-929, 2013.

8. Weiss WA, Aldape K, Mohapatra G, Feuerstein BG and Bishop JM: Targeted expression of MYCN causes neuroblastoma in transgenic mice. EMBO J 16: 2985-2995, 1997.

9. Maris JM,Hogarty MD, Bagatell R and Cohn SL: Neuroblastoma. Lancet 369: 2106-2120, 2007.

10. Pugh TJ, Morozova O, Attiyeh EF, Asgharzadeh S, Wei JS, Auclair D, Carter SL, Cibulskis K, Hanna M, Kiezun A, et al: The genetic landscape of high-risk neuroblastoma. Nat Genet 45: 279-284, 2013.

11. Molenaar JJ, Koster J, Zwijnenburg DA, van Sluis P, Valentijn LJ, van der Ploeg I, Hamdi M, van Nes J, Westerman BA, van Arkel J, et al: Sequencing of neuroblastoma identifies chromothripsis and defects in neuritogenesis genes. Nature 483: 589-593, 2012

12. Cheung NK, Zhang J, Lu C, Parker M, Bahrami A, Tickoo SK, Heguy A, Pappo AS, Federico S, Dalton J, et al; St Jude Children's Research Hospital-Washington University Pediatric Cancer Genome Project: Association of age at diagnosis and genetic mutations in patients with neuroblastoma. JAMA 307: 1062-1071, 2012

13. Poremba C, Scheel C, Hero B, Christiansen H, Schaefer KL, Nakayama Ji, Berthold F, Juergens H, Boecker W and Dockhorn-Dworniczak B: Telomerase activity and telomerase subunits gene expression patterns in neuroblastoma: A molecular and immunohistochemical study establishing prognostic tools for fresh-frozen and paraffin-embedded tissues. J Clin Oncol 18: 2582-2592, 2000.

14. Brodeur GM and Bagatell R: Mechanisms of neuroblastoma regression. Nat Rev Clin Oncol 11: 704-713, 2014.

15. Mac SM, D'Cunha CA and Farnham PJ: Direct recruitment of $\mathrm{N}$-myc to target gene promoters. Mol Carcinog 29: 76-86, 2000.

16. Schulte JH, Horn S, Otto T, et al: MYCN regulates oncogenic MicroRNAs in neuroblastoma. Int J Cancer 122: 699-704, 2008.

17. Cobrinik D, Ostrovnaya I, Hassimi M, Tickoo SK, Cheung IY and Cheung NK: Recurrent pre-existing and acquired DNA copy number alterations, including focal TERT gains, in neuroblastoma central nervous system metastases. Genes Chromosomes Cancer 52: 1150-1166, 2013.

18. Vandesompele J, De Preter K, Pattyn F, et al: Accurate normalization of real-time quantitative RT-PCR data by geometric averaging of multiple internal control genes. Genome Biol 3: RESEARCH0034, 2002.

19. Bachmann HS, Heukamp LC, Schmitz KJ, et al: Regulatory BCL2 promoter polymorphism $(-938 \mathrm{C}>\mathrm{A})$ is associated with adverse outcome in patients with prostate carcinoma. International journal of cancer. Int J Cancer 129: 2390-2399, 2011.

20. Sadée W, Yu VC, Richards ML, Preis PN, Schwab MR, Brodsky FM and Biedler JL: Expression of neurotransmitter receptors and myc protooncogenes in subclones of a human neuroblastoma cell line. Cancer Res 47: 5207-5212, 1987.

21. Barnes EN, Biedler JL, Spengler BA and Lyser KM: The fine structure of continuous human neuroblastoma lines SK-N-SH, SK-N-BE(2) and SK-N-MC. In Vitro 17: 619-631, 1981.

22. Biedler JL, Helson L and Spengler BA: Morphology and growth, tumorigenicity and cytogenetics of human neuroblastoma cells in continuous culture. Cancer Res 33: 2643-2652, 1973.

23. Killela PJ, Reitman ZJ, Jiao Y, Bettegowda C, Agrawal N, Diaz LA Jr, Friedman AH, Friedman H, Gallia GL, Giovanella BC, et al: TERT promoter mutations occur frequently in gliomas and a subset of tumors derived from cells with low rates of self-renewal. Proc Natl Acad Sci USA 110: 6021-6026, 2013.

24. Lindsey JC, Schwalbe EC, Potluri S, Bailey S, Williamson D and Clifford SC: TERT promoter mutation and aberrant hypermethylation are associated with elevated expression in medulloblastoma and characterise the majority of non-infant SHH subgroup tumours. Acta Neuropathol 127: 307-309, 2014.

25. Papathomas TG, Oudijk L, Zwarthoff EC, Post E, Duijkers FA, van Noesel MM, Hofland LJ, Pollard PJ, Maher ER, Restuccia DF, et al: Telomerase reverse transcriptase promoter mutations in tumors originating from the adrenal gland and extra-adrenal paraganglia. Endocr Relat Cancer 21: 653-661, 2014. 